\title{
In situ metabolomics of aldosterone-producing adenomas
}

\author{
Masanori Murakami, ${ }^{1}$ Yara Rhayem, ${ }^{1}$ Thomas Kunzke, ${ }^{2}$ Na Sun, ${ }^{2}$ Annette Feuchtinger, ${ }^{2}$ \\ Philippe Ludwig, ${ }^{1}$ Tim Matthias Strom, ${ }^{3,4}$ Celso Gomez-Sanchez, ${ }^{5}$ Thomas Knösel, ${ }^{6}$ \\ Thomas Kirchner, ${ }^{6}$ Tracy Ann Williams,, ${ }^{1,7}$ Martin Reincke, ${ }^{1}$ Axel Karl Walch, ${ }^{2}$ and Felix Beuschlein ${ }^{1,8}$ \\ ${ }^{1}$ Medizinische Klinik und Poliklinik IV, Klinikum der Universität, Ludwig-Maximilians-Universität München, Munich, \\ Germany. ${ }^{2}$ Research Unit Analytical Pathology and ${ }^{3}$ Institute of Human Genetics, Helmholtz Zentrum München, German \\ Research Center for Environmental Health (GmbH), Neuherberg, Germany. ${ }^{4}$ Institute of Human Genetics, Technische \\ Universität München, Munich, Germany. ${ }^{5}$ Division of Endocrinology, G.V. (Sonny) Montgomery VA Medical Center, and \\ the University of Mississippi Medical Center, Jackson, Mississippi, USA. ${ }^{6}$ Institute of Pathology, Ludwig-Maximilians- \\ Universität München, Munich, Germany. ${ }^{7}$ Division of Internal Medicine and Hypertension, Department of Medical Sciences \\ University of Turin, Turin, Italy. ${ }^{8}$ Klinik für Endokrinologie, Diabetologie und Klinische Ernährung, Unviersitätsspital Zürich, \\ Zurich, Switzerland.
}

\begin{abstract}
Recent genetic examinations and multisteroid profiles have provided the basis for subclassification of aldosterone-producing adenomas (APAs). The objective of the current study was to produce a comprehensive, high-resolution mass spectrometry imaging (MSI) map of APAs in relation to morphometry, immunohistochemical profiles, mutational status, and clinical outcome. The study cohort comprised 136 patients with unilateral primary aldosteronism. Matrix-assisted laser desorption/ionization-Fourier transform-ion cyclotron resonance MSI was conducted, and metabolite profiles were analyzed with genotype/phenotype information, including digital image analysis from morphometry and IHC of steroidogenic enzymes. Distinct molecular signatures between KCNJ5- and CACNA1D-mutated APAs with significant differences of 137 metabolites, including metabolites of purine metabolism and steroidogenesis, were observed. Intratumor concentration of 18-oxocortisol and 18-hydroxycortisol were inversely correlated with the staining intensity of CYP11B1. Lower staining intensity of CYP11B1 and higher levels of 18-oxocortisol were associated with a higher probability of complete clinical success after surgery. The present study demonstrates distinct metabolomic profiles of APAs in relation to tumor genotype. In addition, we reveal an inverse correlation between cortisol derivatives and CYP11B1 and the impact of 18-oxocortisol and CYP11B1 on clinical outcome, which provides unprecedented insights into the pathophysiology, clinical features, and steroidogenesis of APAs.
\end{abstract}

Authorship note: MM and YR contributed equally to this work and are co-first authors.

Conflict of interest: The authors have declared that no conflict of interest exists

Copyright: (c) 2019, American Society for Clinical Investigation.

Submitted: May 17, 2019

Accepted: August 1, 2019

Published: September 5, 2019

Reference information: JCI Insight. 2019;4(17):e130356.

https://doi.org/10.1172/jci.

insight.130356.

\section{Introduction}

Primary aldosteronism (PA) - the excessive secretion of aldosterone independent of angiotensin II is regarded as the most prevalent form of endocrine hypertension. Patients with PA can be subdivided into those affected by bilateral hyperplasia and those with the unilateral form of the disease that usually relates to the presence of an aldosterone-producing adenoma (APA) of the adrenal cortex. Clinically, PA is characterized by high blood pressure, electrolyte disturbances, and the development of excessive cardiovascular complications compared with essential hypertension $(1,2)$. The diagnosis of APA is of particular importance because this condition can be cured by unilateral adrenalectomy. However, the likelihood of complete clinical success after surgery varies widely between clinical centers and individual patients (3).

IHC with newly available specific monoclonal antibodies has been proved very useful in providing a histopathological diagnosis of PA by quantification and localization of steroidogenic enzymes, such as aldosterone synthase (cytochrome P450, family 11, subfamily B, polypeptide 2: CYP11B2) $(4,5)$. Further in-depth molecular characterization has become possible more recently by means of high-throughput "omics" technologies, including exome sequencing, transcriptomics (6), and methylomics $(7,8)$. In particular, exome sequencing has contributed to uncovering genes (including KCNJ5, ATP1A1, ATP2B3, CACNA1D, CACNA1H, and $C T N N B 1)$ in which specific gain-of-function mutations result in autonomous aldosterone production (9-13). 
In addition to these genomic studies, information from multisteroid analyses have specified and extended the endocrine spectrum of PA $(14,15)$. Based on 24-hour urine steroid profiles, it has been demonstrated that a substantial subgroup of patients with PA are characterized by increased glucocorticoid output in addition to aldosterone excess (15). Furthermore, steroid profiles from adrenal venous and peripheral plasma from APA patients were shown to carry information on the presence of somatic mutations of the adenoma (14).

The ability of mass spectrometry imaging (MSI) to localize biomolecules in tissues without prior knowledge of their presence has recently led to a rapid and substantial impact on translational and clinical research by uncovering molecular changes associated with disease. A broad spectrum of analytes, including proteins, protein modifications, peptides, drugs and their related metabolites, endogenous cell metabolites, lipids, contrast agents, and other molecules, have been made accessible by this in situ technique (16). In addition to the intensities of mass spectrometry, MSI studies by high-resolution matrix-assisted laser desorption/ionization-Fourier transform-ion cyclotron resonance (MALDI-FT-ICR) permit correlations with the spatial information conveyed by conventional histology. This technique has recently allowed us to refine the functional anatomy of the human adrenal gland, which supports the usefulness of these analyses for steroid-producing tissues also (17).

Here, we report what we believe is the first comprehensive MSI study of APAs including genotype/ phenotype information and digital image analysis from morphometry and IHC of steroidogenic enzymes to gain further insights into APA pathogenesis and clinical outcome of patients with this condition.

\section{Results}

Demographics. Clinical characteristics of the 136 patients with APA (77 men and 59 women, age at adrenalectomy $50.7 \pm 10.0$ years) are described and stratified by clinical outcome in Table 1 . Clinical outcomes were evaluated based on the recently established international consensus on surgery outcomes for unilateral PA (3). For 132 patients on which genetic annotations were available, the following APA genotypes were present: $53(40.2 \%)$ without a recurrent mutation in exome or Sanger sequencing, referred to as wild-type; 49 (37.1\%) KCNJ5 mutations; 9 (6.8\%) ATP1A1 mutations; 6 (4.6\%) ATP2B3 mutations; 11 (8.3\%) $C A C$ NA1D mutations; 2 (1.5\%) CTNNB1 mutations; and 2 (1.5\%) PRKACA mutations.

Morphometry and genotype correlation. Following the described workflow (Supplemental Figure 1; supplemental material available online with this article; https://doi.org/10.1172/jci.insight.130356DS1), first, H\&E sections from the TMA, consisting of samples from 136 patients with APA, were analyzed by morphometry as a method of computer-assisted pattern recognition. Among data from color and shape parameters of nucleus and cytoplasm, the standard deviation of nuclear size and brightness of cytoplasm was significantly correlated with genotypes. Higher standard deviation of nuclear size was observed in KCNJ5-mutated APAs in comparison with wild-type, ATP1A1-, and CACNA1D-mutated APAs (adjusted $P<0.001$; Figure 1, $\mathrm{A}$ and B). Higher brightness of cytoplasm was also observed in $K C N J 5$-mutated APAs in comparison with wild-type and ATP1A1-mutated APAs (adjusted $P=0.022$; Supplemental Figure 2, A and B).

Steroidogenic enzyme expression and genotype/phenotype correlation. In addition to morphometry, IHC of steroidogenic enzymes, including CYP11B1, CYP11B2, CYP17A1, HSD3B1, and HSD3B2, was performed, and staining intensities were assessed by digital image analysis. Correlations between immunohistochemical staining and steroidogenic enzyme expression are summarized in Table 2, which indicates a variety of significant correlations. For example, staining intensities of CYP11B1 were positively correlated with those of CYP17A1 $\left(r_{s}=\right.$ 0.515 , and $P<0.001)$ and HSD3B1 $\left(r_{s}=0.355\right.$, and $\left.P<0.001\right)$ but inversely correlated with those of CYP11B2 $\left(r_{s}=-0.414\right.$, and $\left.P<0.001\right)$ and HSD3B2 $\left(r_{s}=-0.343\right.$, and $\left.P<0.001\right)$. Relationships between staining intensities of steroidogenic enzymes and genotypes were further examined. According to these analyses, staining intensities of CYP11B1 in KCNJ5-mutated APAs were significantly lower in comparison with wild-type $(P=0.004)$ without further relevant genotype-dependent variations (Figure 1C and Supplemental Table 1). Staining intensities for CYP11B2 were elevated in ATP1A1-mutated APAs in comparison with $K C N J 5$-mutated $(P<0.001)$ and CACNA1D-mutated $(P=0.021)$ APAs (Figure 1D).

Metabolome profiles in correlation to genotypes. Following MALDI-FT-ICR MSI analysis, unguided hierarchical clustering analysis of the whole data set failed to distinguish unequivocal clusters (Supplemental Figure 3A). Based on these initial heterogeneous profiles, comparisons of individual genotype pairs were performed. These analyses unraveled distinct metabolome profiles between KCNJ5- and CACNA1D-mutated APAs (Figure 2A), which were supported on the basis of ortho-PLSDA clustering (Figure 2B). Among approximately $371 \mathrm{~m} / z$ species, which were extracted by matrix cleaning, over 
Table 1. Clinical characteristics of the 136 patients with APA included in the analysis, with stratification by clinical outcome

n Total cohort Complete Partial Absent Pvalue Complete Complete Partial Complete Complete Complete vs. vs. vs. vs. partial plus vs. partial absent absent plus partial vs. absent absent absent



Data are mean (SD), $n(\%), n / N(\%)$, or median (IQR). All variables refer to preoperative data unless stated otherwise. $P$ values of less than 0.05 were considered significant and are shown in bold. Adrenal nodule refers to diameter of largest nodule at pathology. Lateralisation index: ([aldosterone]/[cortisol] dominant adrenal vein $/\left([\right.$ aldosterone $\left.] /[\text { cortisol }]_{\text {nondominant adrenal vein }}\right)$. Contralateral ratio: ([aldosterone $] /[\text { cortisol }]_{\text {nondominant adrenal vein }} /\left([\right.$ aldosterone $\left.] /[\text { cortisol }]_{\text {peripheral vein }}\right)$. NA, not applicable; SBP, systolic blood pressure; DBP, diastolic blood pressure; ARR, aldosterone/renin ratio; DST, dexamethasone suppression test.

a mass range of 50 to $1000 \mathrm{~m} / z, 137$ metabolites were significantly different (adjusted $P<0.05$ ) in KCNJ5- and CACNA1D-mutated APAs. Furthermore, pathway analysis based on significantly altered metabolites was performed and revealed activation of purine metabolism in KCNJ5-mutated APAs (pathway impact $=0.13, P<0.001$, and FDR $<0.001$ ). Specifically, higher intensities of metabolites, such as $\operatorname{AMP}(m / z$ 328.045), $\operatorname{ADP}(m / z 408.0115)$, and D-Ribulose 5-phosphate $(m / z 211.001)$, were 
A

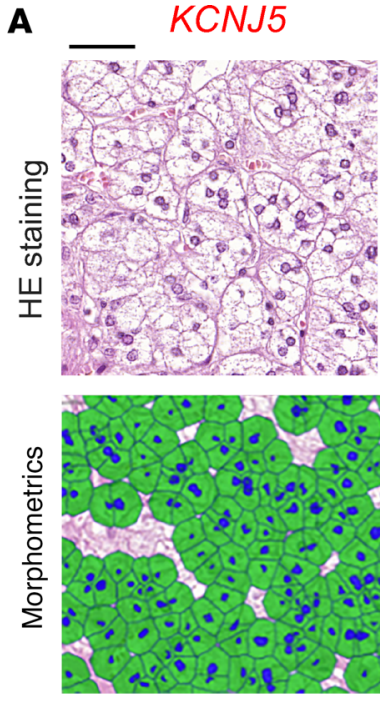

nucleus

\section{CACNA1D}
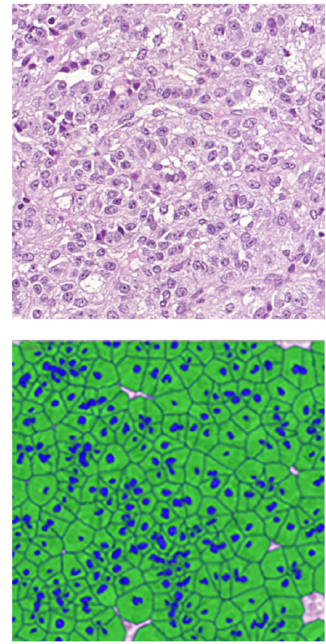

cytoplasm

\section{c}

CYP11B1

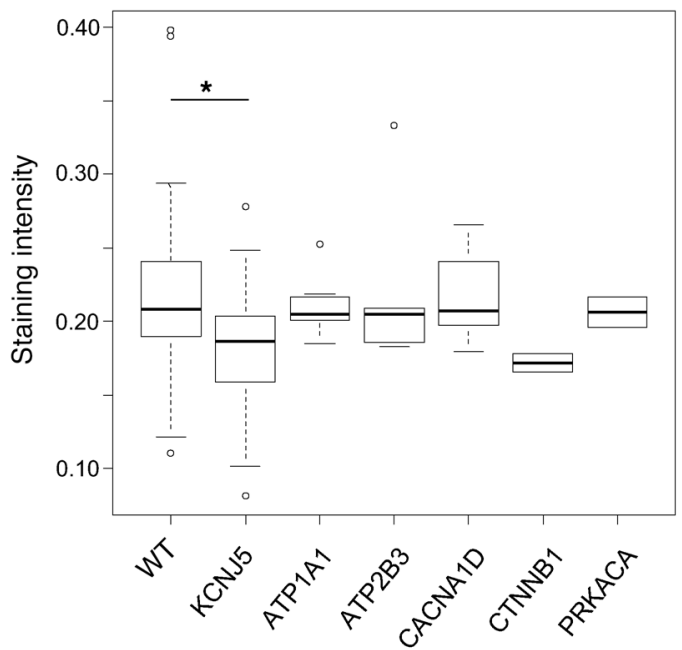

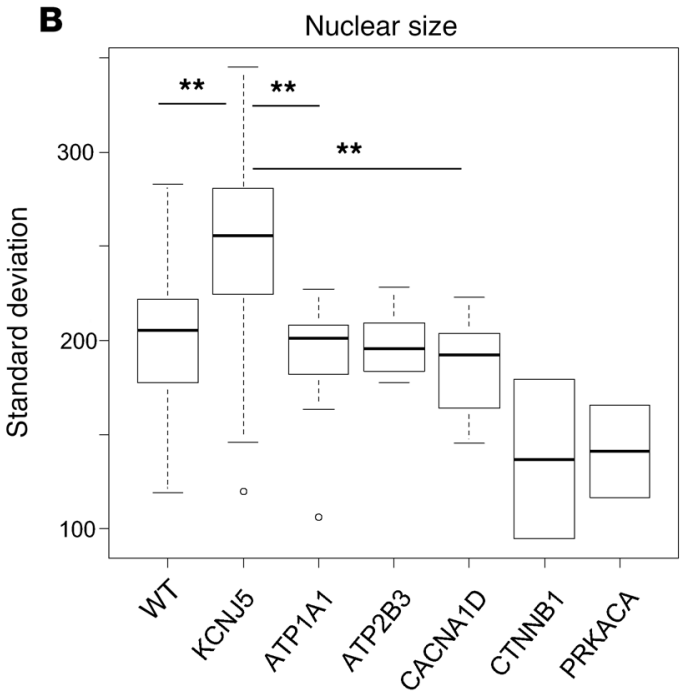

D

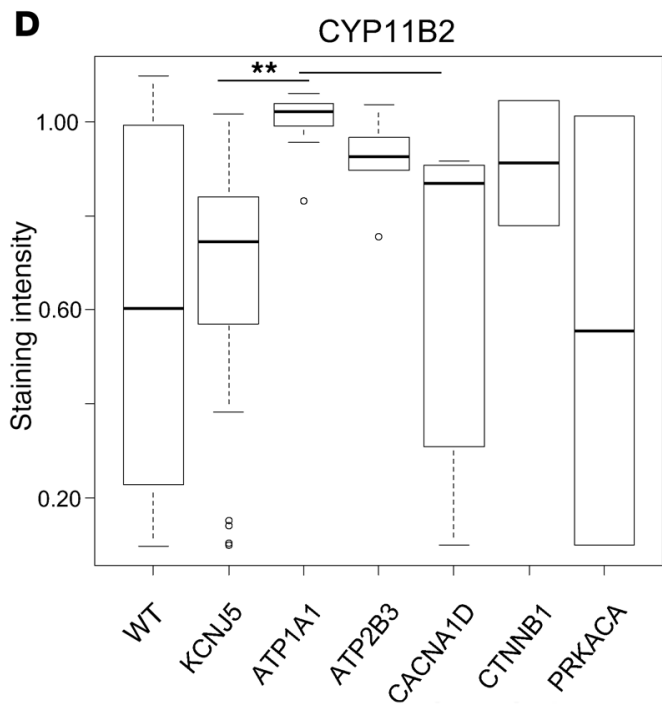

Figure 1. Comparison of morphometric features and genotypes of 132 APA samples. Representative images of H\&E staining and morphometric analysis (A) (scale bar: $50 \mu \mathrm{m}$ ), with standard deviation of nuclear size with respect to genotypes, indicating nuclear variability in KCNJ5-mutated samples (adjusted $P<0.001)$ (B). Quantification of staining intensities for steroidogenic enzymes correlated with mutation status, demonstrating lower CYP11B1 expression in KCNJ5-mutated tumors (C) and higher CYP11B2 expression in ATP1A1-mutated tumors (D). Median intensity of 3 cores from each patient was used for IHC profiles of steroidogenic enzymes. Kruskal-Wallis test was used for statistical analysis. ${ }^{*} P<0.05$; ${ }^{* *} P<0.01$. The box plots depict the minimum and maximum values (whiskers), the upper and lower quartiles, and the median. The length of the box represents the interquartile range.

observed in KCNJ5-mutated APAs (Figure 2C). Considering all genotypes, we could demonstrate a positive correlation between tumor size and AMP $\left(r_{s}=0.198\right.$, and $\left.P=0.021\right)$ and ADP $\left(r_{s}=0.239\right.$, and $P=0.005)$, respectively. A significantly lower abundance of 1-phosphatidyl-D-myo-inositol, which is an intermediate in inositol phosphate metabolism, was observed in KCNJ5-mutated APAs (adjusted $P=0.009$, Figure 2D). Among all measured $m / z$ species, 18-hydroxycortisol $(m / z 377.2)$ and 18-oxocortisol ( $m / z$ 375.185) were successfully annotated metabolites involved in the steroidogenic pathway. Both of these 18-hybrid steroids were found with a higher abundance in KCNJ5-mutated APAs (Figure $2 \mathrm{E})$. Additional clustering analyses focused on other specific parameters, such as the specific $K C N J 5$ mutation status (G151R vs. L168R, Supplemental Figure 3B) and serum cortisol values following $1 \mathrm{mg}$ DST (high of $>1.8 \mu \mathrm{g} / \mathrm{dl}$ vs. low of $\leq 1.8 \mu \mathrm{g} / \mathrm{dl}$ ) (Supplemental Figure $3 \mathrm{C}$ ), were performed, which did not reveal any distinct metabolomic pattern. 
Table 2. Correlations between staining intensities of steroidogenic enzymes

\begin{tabular}{|c|c|c|c|c|c|c|c|c|}
\hline & \multicolumn{2}{|c|}{ CYP11B2 } & \multicolumn{2}{|c|}{ CYP17A1 } & \multicolumn{2}{|c|}{ HSD3B1 } & \multicolumn{2}{|c|}{ HSD3B2 } \\
\hline & $r_{s}$ & $P$ value & $r_{s}$ & $P$ value & $r_{s}$ & $P$ value & $r_{s}$ & $P$ value \\
\hline CYP11B1 & -0.414 & $<0.001$ & 0.515 & $<0.001$ & 0.355 & $<0.001$ & -0.343 & $<0.001$ \\
\hline CYP17A1 & & & - & - & 0.170 & 0.002 & -0.260 & $<0.001$ \\
\hline HSD3B1 & & & & & - & - & -0.178 & 0.002 \\
\hline
\end{tabular}

Correlation of tumor steroid metabolite content and steroidogenic enzyme expression. To explore the correlation between cellular steroid concentration and steroidogenic enzyme distribution in APAs, comparisons between 18-oxocortisol and 18-hydroxycortisol intensities and IHC staining pattern were carried out for all genotypes. Both 18-oxocortisol and 18-hydroxycortisol content were inversely correlated with staining intensities for CYP11B1 $\left(r_{s}=-0.345\right.$, and $P<0.001 ; r_{s}=-0.302$, and $P<0.001$, respectively; Supplemental Table 2$)$ and CYP17A1 $\left(r_{s}=-0.171\right.$, and $P=0.002 ; r_{s}=-0.165$, and $P=0.002$, respectively). In contrast, those of CYP11B2 were positively correlated only with the intensities of 18 -hydroxycortisol $\left(r_{s}=0.107\right.$, and $P=0.039$ ). Following this initial analysis, correlations were restricted to KCNJ5-mutated APAs, in which 18-hybrid steroids were found to be more abundantly distributed. Similar to the whole data set, intensities of both 18-oxocortisol and 18-hydroxycortisol were strongly inversely correlated with staining intensities of CYP11B1 $\left(r_{s}=-0.421\right.$, and $P<0.001 ; r_{s}=-0.367$, and $P<0.001$, respectively $)$ and CYP17A1 $\left(r_{s}=-0.293\right.$, and $P<0.001 ; r_{s}=-0.345$, and $P<0.001$, respectively; Figure 3A and Supplemental Table 2). In addition, a further inverse correlation between 18-hydroxycortisol and HSD3B2 staining was found $\left(r_{s}=-0.247\right.$, and $P=0.009)$. Multiple regression analysis showed a relationship between staining intensities of CYP11B1 and intensities of both 18-oxocortisol $(P<0.001$, Supplemental Table 3$)$ and 18-hydroxycortisol $(P=$ 0.041, Supplemental Table 3), suggesting that cores with lower expression of CYP11B1 were associated with higher intensities of both 18-hybrid steroids (example provided in Figure 3B). In addition, a relationship between staining intensities of CYP17A1 and 18-oxocortisol was evident $(P=0.031$, Supplemental Table 3). Following this initial analysis, we further subdivided APAs in those with low and high CYP17A1 expression. In both groups the inverse correlation between CYP11B1 and 18-oxocortisol remained highly significant $\left(r_{s}=-0.277\right.$, and $P<0.001$; and $r_{s}=-0.306$, and $P<0.001$, respectively; Supplemental Table 4). In addition, a positive correlation between CYP17A1 and 18-oxocortisol $\left(r_{s}=0.213\right.$, and $P=0.005$, Supplemental Table 4) was evident in tissues with an overall lower-than-median intensity of CYP17A1 (examples provided in Figure 3C), while an inverse correlation between these parameters was found in tissues with a higher-than-median intensity of CYP17A1 $\left(r_{s}=-0.226\right.$, and $P=0.003$; Supplemental Table 4 and example provided in Supplemental Figure 4).

Impact of IHC and metabolic profiles on clinical outcome. Metabolomic parameters and IHC-based profiles were further correlated with clinical annotations at baseline and during follow-up after surgical therapy. According to these studies, intensities of 18-oxocortisol were positively correlated with tumor size $\left(r_{s}=0.218\right.$, and $P=0.011)$ and inversely correlated with $\mathrm{DBP}\left(r_{s}=-0.232\right.$, and $\left.P=0.007\right)$ and 24-hour urinary free cortisol $\left(r_{s}=-0.365\right.$, and $\left.P=0.016\right)$. In addition, intensities of 18 -hydroxycortisol were inversely correlated with DBP $\left(r_{s}=-0.173\right.$, and $\left.P=0.044\right)$. Notably, a strong correlation between plasma 18-oxocortisol and tissue 18-oxocortisol $\left(r_{s}=0.321\right.$, and $\left.P=0.009\right)$ and a weak correlation between plasma and tissue 18-hydroxycortisol $\left(r_{s}=0.209\right.$, and $\left.P=0.095\right)$ were evident. With regard to clinical outcome, patients with complete clinical success showed lower staining intensities of CYP11B1 in comparison with those with partial clinical success $(P=0.006)$ and absent clinical success $(P<0.001$, Figure 4A and Supplemental Table 5). Conversely, there was no significant correlation between staining intensities of CYP11B2 and clinical outcome $(P=0.449$, Figure 4B). In addition, APAs from patients with absent clinical success showed lower staining intensities of HSD3B2 in comparison with those with partial clinical success $(P=0.043$, Supplemental Table 5). Furthermore, patients with complete clinical success had higher tumor intensities of 18-oxocortisol in comparison with those with partial clinical success $(P=0.036$, Figure $4 C)$ and those with partial plus absent clinical success $(P=0.015$, Figure $4 \mathrm{D})$. To clarify whether IHC and metabolic profiles are independently associated with 
A

KCNJ5 CACNA1D

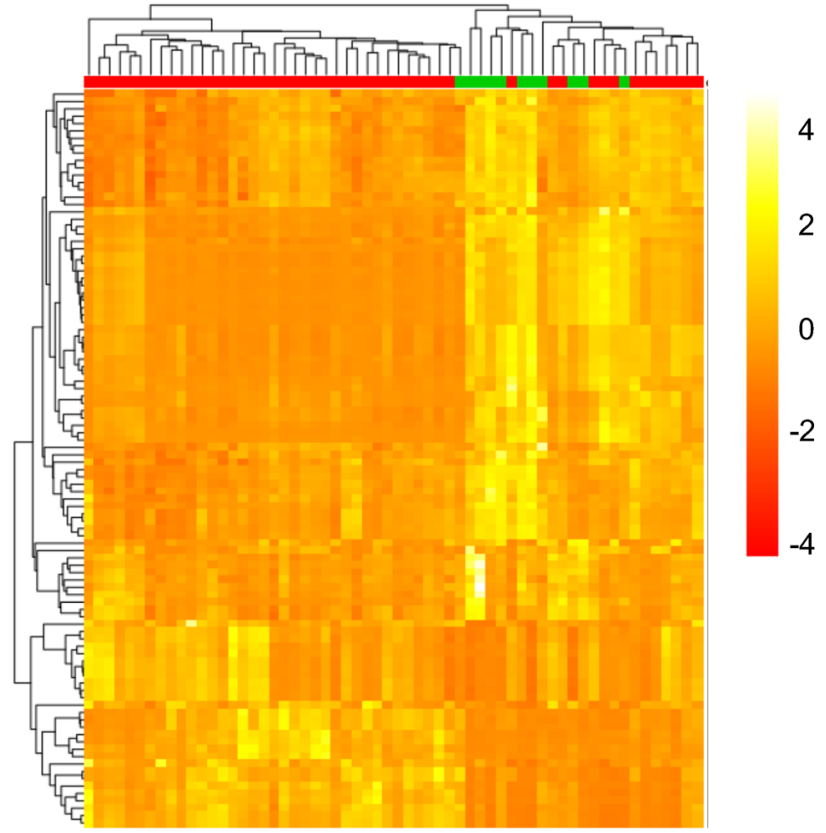

Unguided hierarchical cluster analysis

C

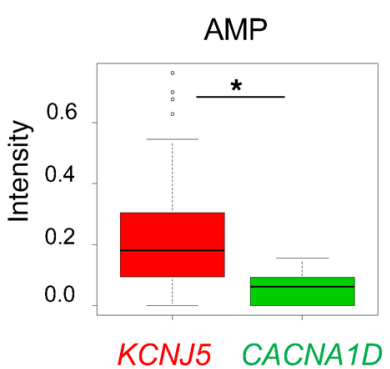

E

18-oxocortisol

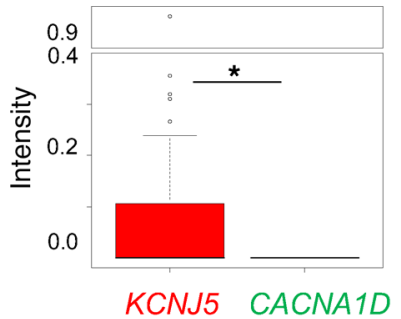

Purine metabolism

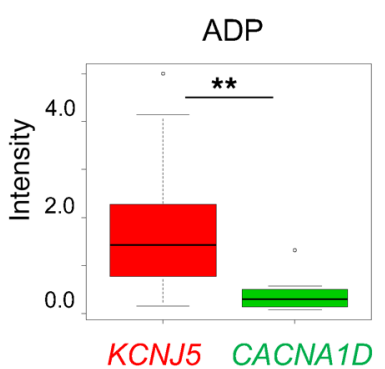

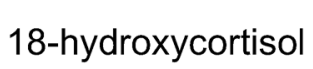

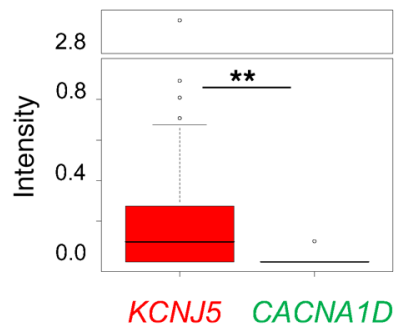

B $\quad \mathrm{KCNJ5}$

CACNA1D

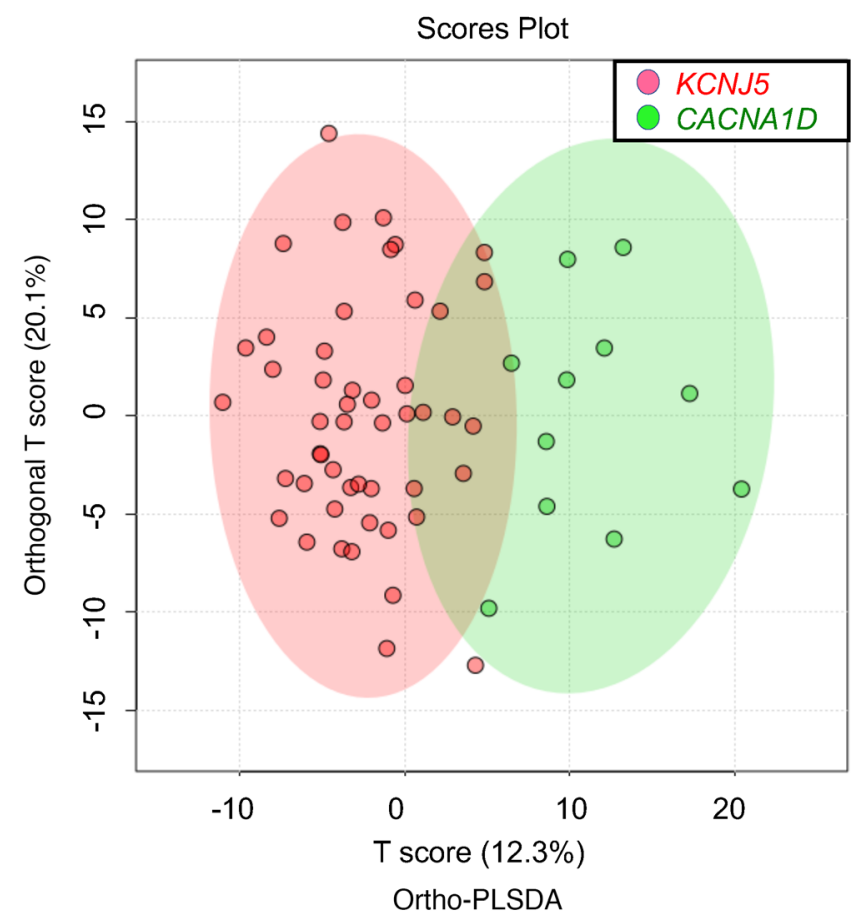

D-Ribulose 5-phosphate

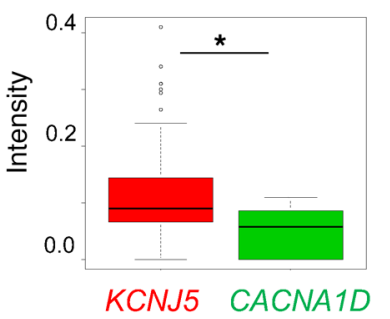

D

1-Phosphatidyl-D-myo-inositol

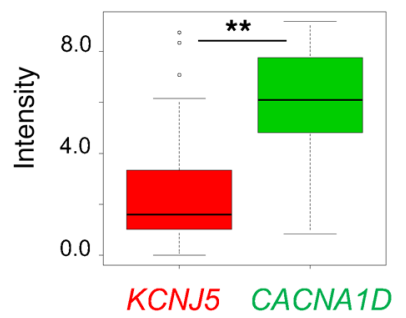

Figure 2. Metabolome features of 60 APA samples. Unguided hierarchical clustering analysis (A) and orthogonal partial least squares discriminant analysis (ortho-PLSDA) (B) of metabolome profiles between APAs with KCNJ5 and CACNA1D mutations. (C) Shown are significant differences in metabolites of the purine metabolism and intensities of adenosine monophosphate (AMP), adenosine diphosphate (ADP), and D-Ribulose 5-phosphate between KCNJ5- and CACNA1D-mutated APAs (adjusted $P=0.010,0.003$, and 0.047, respectively). (D) Intensities of 1-phosphatidyl-D-myo-inositol between KCNJ5- and CACNA1D-mutated APAs (adjusted $P=0.009$ ). ${ }^{*}$ adjusted $P<0.05$; ${ }^{*}$ adjusted $P<0.01$. (E) Significant changes in intensities between KCNJ5- and CACNA1D-mutated APAs of 18 -oxocoritsol $(P=0.020)$ and 18 -hydroxycortisol $(P<0.001)$. Mann-Whitney $U$ test was used for statistical analysis. ${ }^{*} P<0.05 ;{ }^{*} P<0.01$. The box plots depict the minimum and maximum values (whiskers), the upper and lower quartiles, and the median. The length of the box represents the interquartile range. 
A CYP11B1

18-oxocortisol 18-hydroxycortisol

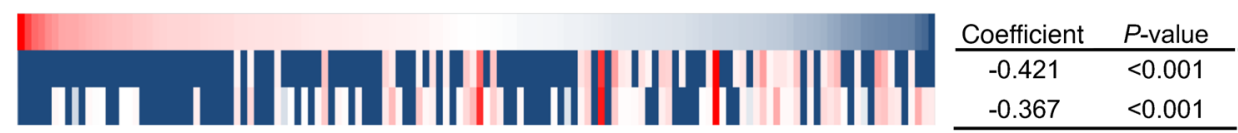

\section{CYP11B2}

18-oxocortisol 18-hydroxycortisol
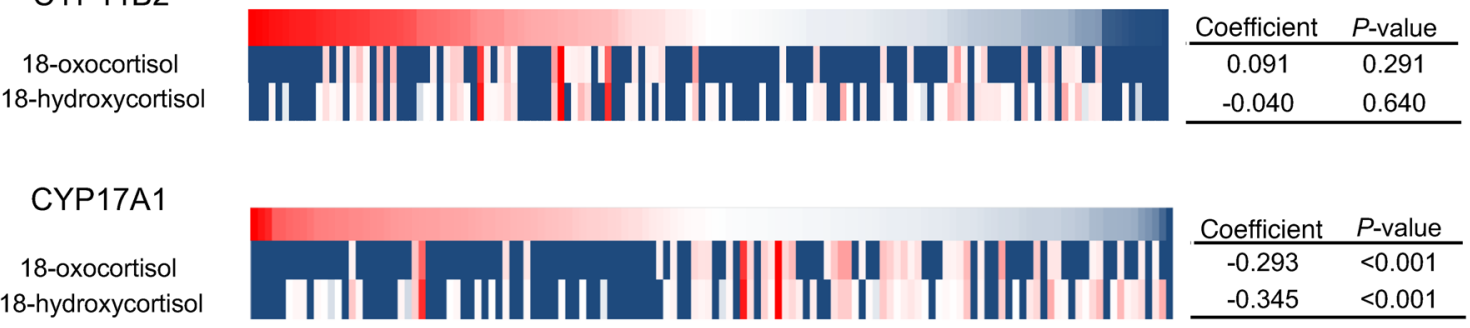

\section{HSD3B1}

18-oxocortisol 18-hydroxycortisol

\section{HSD3B2}

18-oxocortisol

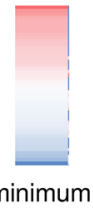

B
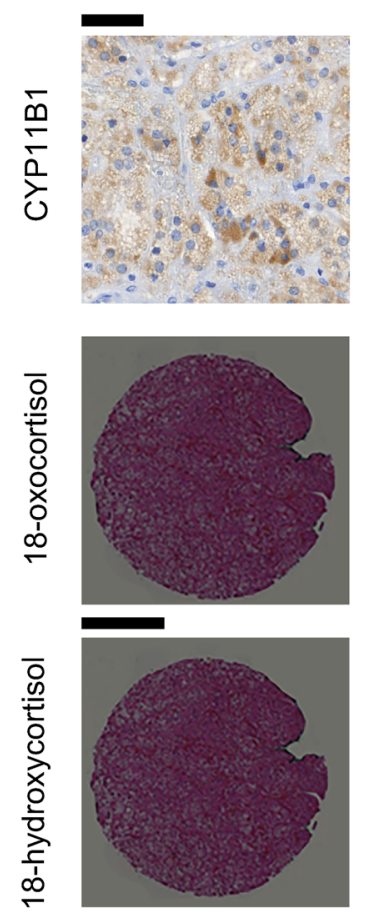

C
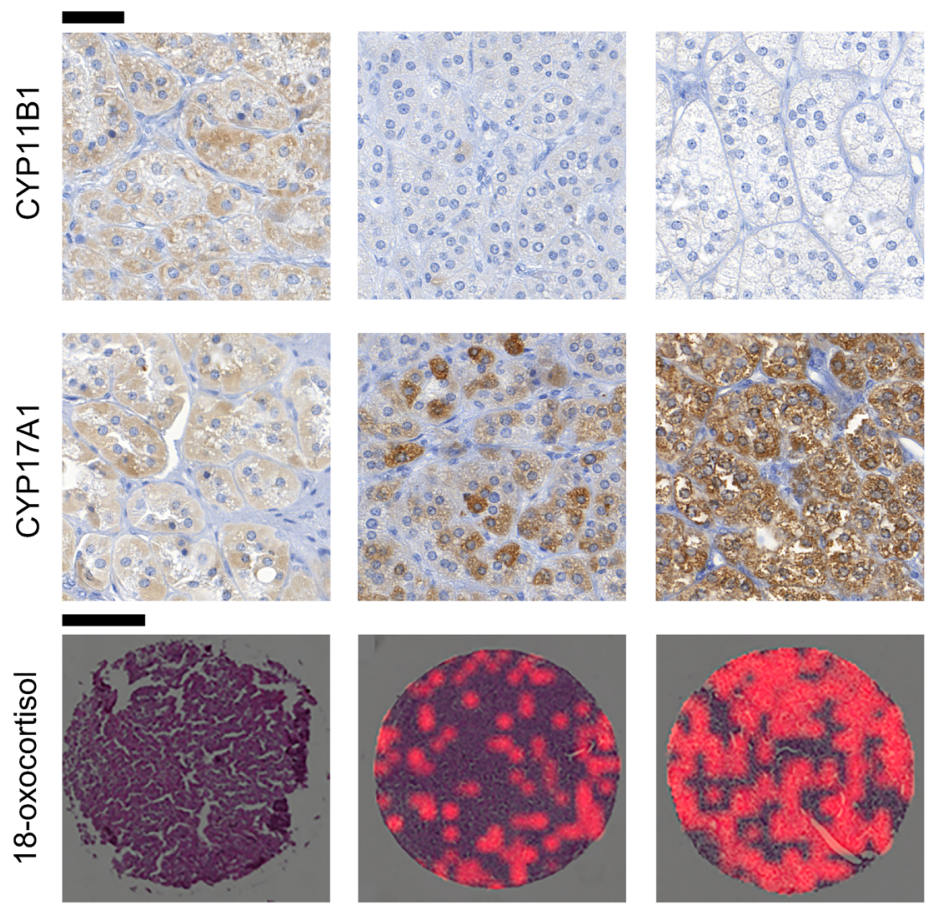

$\max$

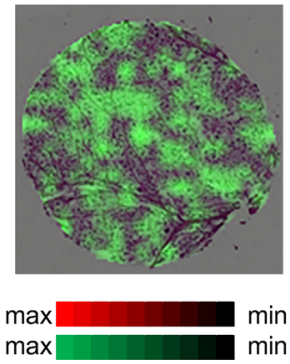

Figure 3. Correlations between IHC of steroidogenic enzymes and metabolic profiles of 18-hydroxycortisol and 18-oxocortisol. (A) Heatmap visualizations of relationships between IHC and 18-oxocortisol and 18-hydroxycortisol in 49 KCNJ5-mutated APAs with results of Spearman's rank correlation coefficient. (B) Representative images of 18-oxocortisol (red) and 18-hydroxycortisol (green) expression from cores with high and low staining intensity of CYP11B1. (C) Comparison of immunohistochemistry of CYP11B1 and CYP17A1 and 18-oxocortisol expression (red) in cores with lower-than-median expression of CYP17A1. Scale bars: 500 (metabolites), $50 \mu \mathrm{m}$ (IHC). 
A

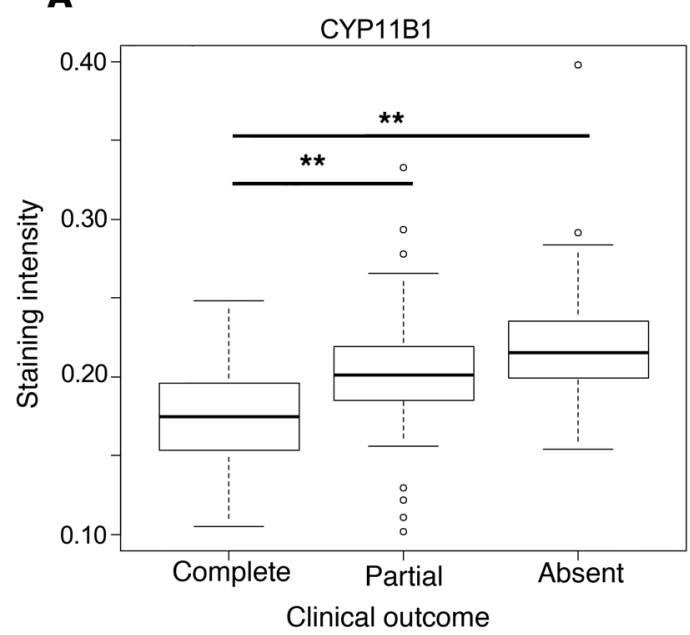

C

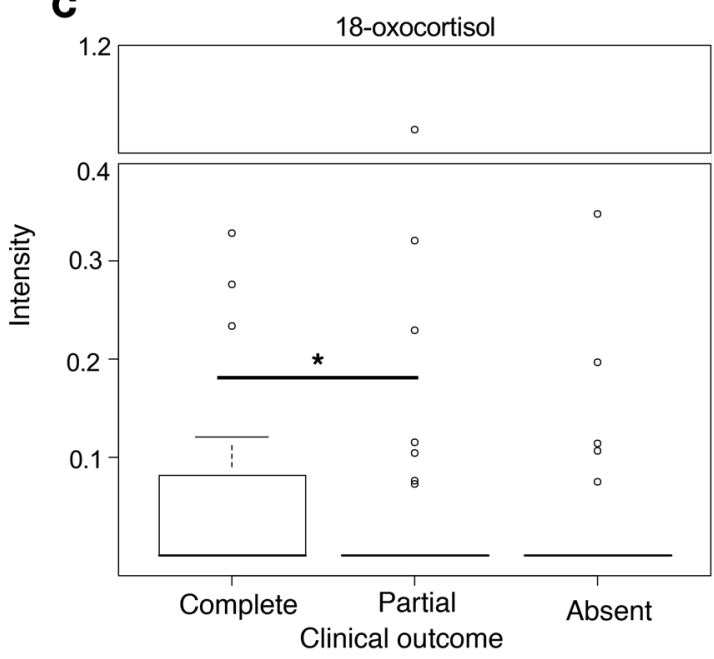

B

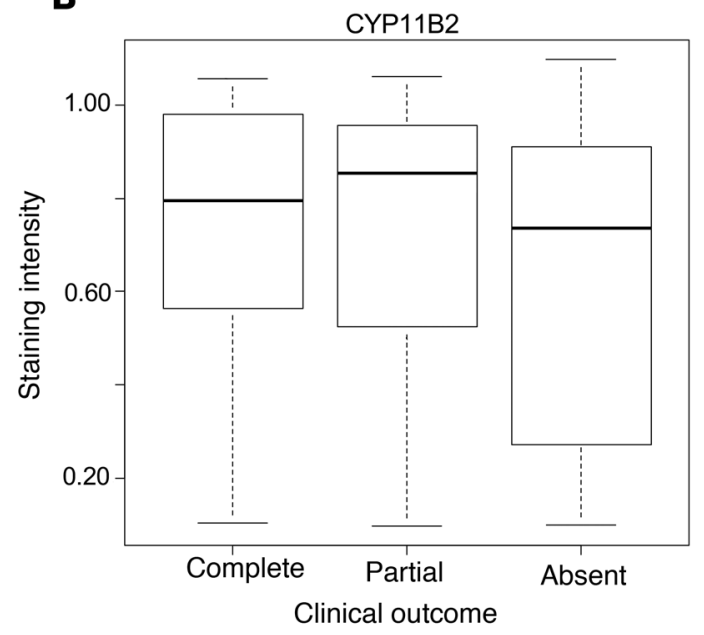

D

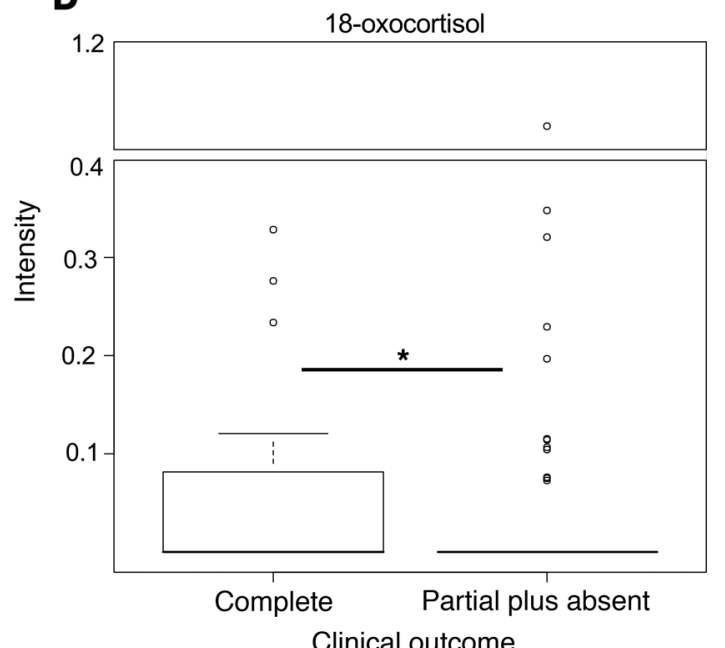

Figure 4. IHC and metabolic profiles on clinical outcome of 136 patients with APA. Staining intensities of steroidogenic enzymes with regard to clinical outcome: CYP11B1 $(P<0.001)(\mathbf{A})$ and CYP11B2 $(P=0.449)(B)$. Intensities of 18 -oxocortisol in relation to clinical outcome: comparison among complete, partial, and absent success in clinical outcome $(P=0.041)(C)$ and that between complete and partial plus absent success in clinical outcome $(P=0.015)$ (D). Intensities of 3 cores from each patient were classified into highest, median, and lowest intensity. Median intensity of 3 cores from each patient was used for analysis. Mann-Whitney $U$ test and Kruskal-Wallis test were used for statistical analysis. ${ }^{*} P<0.05 ;{ }^{*} P<0.01$. The box plots depict the minimum and maximum values (whiskers), the upper and lower quartiles, and the median. The length of the box represents the interquartile range.

clinical outcome, logistic regression analyses were performed. We thereby identified factors independently associated with complete clinical success by comparing the proportion of patients with complete clinical success with those with partial and absent clinical success combined. Following this approach, complete clinical success was associated with lower age, lower BMI, and lower staining intensities of CYP11B1 or higher intensities of 18-oxocortisol (Table 3), while stepwise regression analysis rejected clinical parameters such as maximal tumor size, lateralization index, and KCNJ5 mutation status. Younger age and lower staining intensities of CYP11B1 or higher staining intensities of HSD3B2 were independently associated with clinical benefit when the proportion of patients with complete and partial clinical success combined was compared with the proportion of those with absent clinical success (Table 3). In a comparison of complete with absent success, younger age and lower staining intensities of CYP11B1 were associated with clinical success (Table 3), while stepwise regression rejected clinical parameters such as BMI, contralateral ratio, and KCNJ5 mutation status. Logistic regression analyses were also performed to identify further metabolomic factors independently associated with clinical success. As shown in Supplemental Tables 6, metabolites were associated with complete clinical success in comparison with those with partial and absent clinical success combined. 
Table 3. Multivariate logistic regression analyses including IHC and metabolic profiles

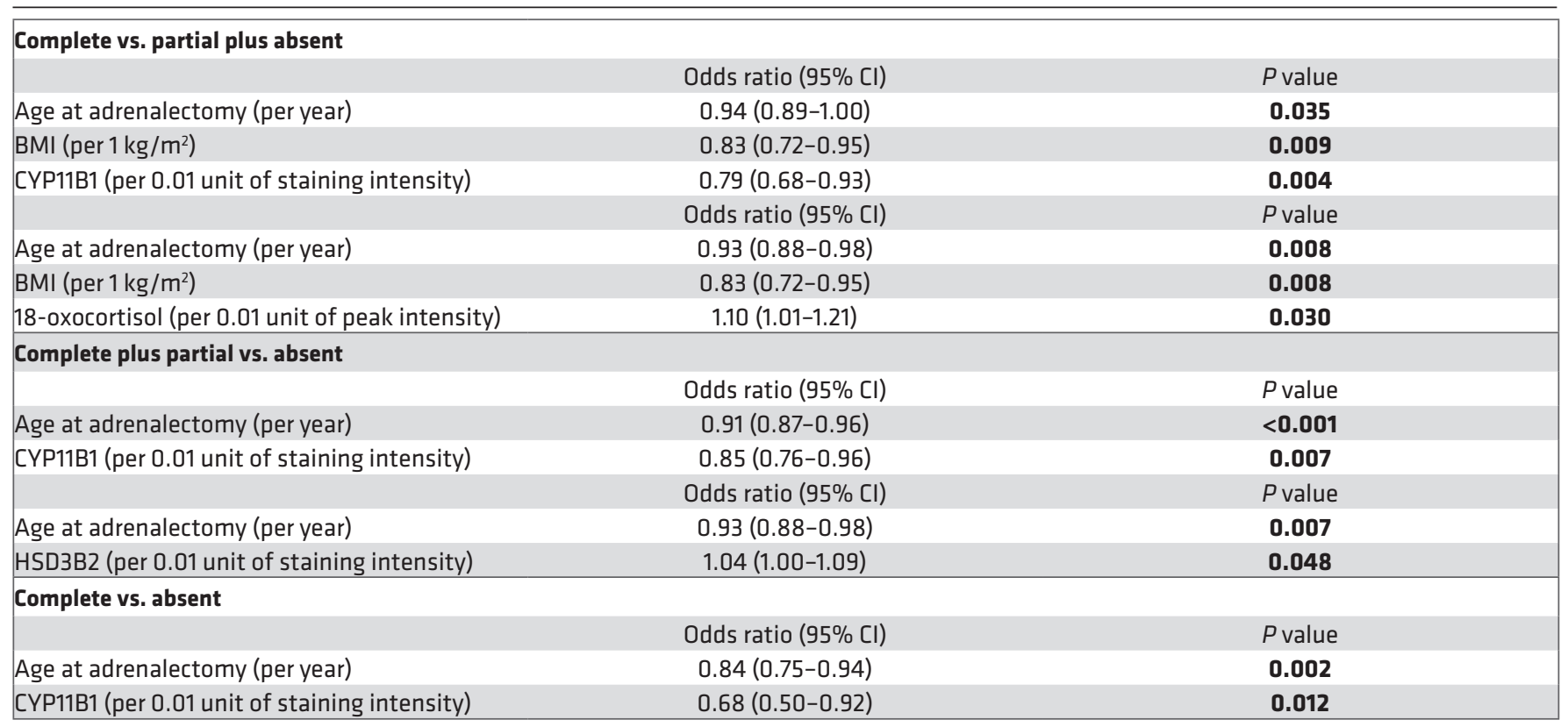

$P$ values less than 0.05 were considered significant and are shown in bold.

\section{Discussion}

Herein, we report an integrated approach of MALDI metabolite imaging, morphometry, and quantification of steroid enzyme expression in APAs to allow deep insights into pathophysiological and clinical features of APAs and associated steroidogenesis. Based on these analyses, we can provide findings in pathways and intratumor steroid enzymes in a genotype-dependent manner that had not been appreciated before and that correlate with clinical outcome.

One of the important findings of our report relates to morphometry, which provides a rich source of information that can be assessed in a quantitative manner. Classification of zona glomerulosa-like (ZG-like) adenomas with small, compact cells, with high nuclear/cytoplasmic ratio and moderate amount of lipid, and zona fasciculata-like (ZF-like) adenomas with large, lipid-laden clear cells, with round to oval vesicular nuclei, has been described $(18,19)$. KCNJ5-mutated APAs were suggested to be composed mainly of ZF-like cells, while ATP1A1- and CACNA1D-mutated APAs more frequently presented a ZG-like phenotype $(20,21)$. However, quantitative assessment of these observations had been difficult. Our morphometry analysis, which is a method of computer-assisted pattern recognition, enabled us to quantify color and shape parameters independent of observer bias. Among parameters with significant differences between genotypes in our analysis, higher brightness of the cytoplasm in KCNJ5-mutated APAs likely reflects the lipid-rich cytoplasm of ZF-like cells. Standard deviation of nuclear size was also found as a parameter in correlation with genotypes. A previous study indicated that KCNJ5-mutated APAs include atypical cells with large nuclei, which were absent in ATP1A1- and $C A C N A 1 D$-mutant APAs (21). It is possible that the finding from the current unsupervised digital image analysis is consistent with this observation. Although pathophysiological significance has to be elucidated, it is conceivable that changes in nuclear variability could reflect difference of cellular origins or differentiation between those genotypes.

Digital image analysis further provided important information on the quantification of steroidogenic enzyme expression. Specifically, staining intensities of CYP11B2 were higher in ATP1A1-mutated APAs, in comparison with those with KCNJ5 mutations, which is consistent with findings from a previous study (20). Notably, we could further demonstrate significant mutual correlations between steroidogenic enzyme expressions. Inverse correlations between staining intensities of CYP11B2 and those of CYP11B1 and CYP17A1 in APAs might reflect the contrasted features of ZG-like cells, which include CYP11B2 ${ }^{+}$cells, and ZF-like cells, which include CYP11B1 ${ }^{+}$and $\mathrm{CYP} 17 \mathrm{~A} 1^{+}$cells. 
Furthermore, our result showed a positive correlation between staining intensities of HSD3B2 and CYP11B2 in APAs, which is consistent with the features of ZG, in which coexpression of HSD3B2 and CYP11B2 has been reported (22).

Previous studies have aimed to classify APAs with regard to genotypes using various approaches, including clinical (23), histopathological $(20,21)$, steroidogenic (14), and molecular parameters $(8,24)$. The MALDI-MSI data, provided herein, add findings directed toward metabolites distributed in tumor tissues. Compared with the high proportion of KCNJ5 mutation (24\%-41\% in Western countries) (24-28), the incidence of other mutations, such as ATP1A1 (5.3\%) and CACNA1D (9.3\%), is rather low (23). This fact is likely to have contributed to a less distinct genotype/phenotype correlation. In addition, heterogeneous profiles of tumors without recurrent mutations, which were observed in the initial unguided hierarchical clustering analysis, might have impeded the unraveling of distinct metabolomic patterns within the whole cohort. However, our cohort included 11 CACNA1D-mutated APAs, which is the highest number reported to date used for molecular analysis of APAs and allowed discrimination of metabolome patterns between KCNJ5- and CACNA1D-mutated APAs. Among differentially present metabolites, higher intensities of 18-oxocortisol and 18-hydroxycortisol in KCNJ5-mutated APAs were concordant with findings from a previous study on plasma steroid concentrations (14).

Moreover, pathway analysis revealed significant changes in purine metabolism and promotion in purine synthesis in KCNJ5-mutated APAs. Purine nucleotides are crucial for providing cellular energy and affect intracellular signaling (29). Based on clinical observations, it is well appreciated that APAs with KCNJ5 mutation are characterized by a larger tumor size (23), which is recapitulated in the current data set with significantly larger KCNJ5 tumors in comparison with those with CACNA1D mutations (data not shown). Considering that an increase in purine synthesis might reflect the promotion of cell cycle and proliferation, the observed larger tumor size and promotion of purine synthesis in APAs with KCNJ5 mutation could be connected by means of active cell proliferation. In fact, considering all genotypes, we were able to demonstrate a positive correlation between tumor size and tumor AMP and ADP content, respectively. Furthermore, purines, including ATP, ADP, and AMP, have been shown to stimulate steroidogenesis in bovine adrenal cells (30) potentially by inhibition of noninactivating $\mathrm{K}^{+}$current, thereby modulating the resting membrane potential (31) and increasing intracellular $\mathrm{Ca}^{2+}$ levels (32). Therefore, it is prudent to speculate that abundant purines in KCNJ5-mutated APAs could contribute to or modify steroidogenesis as auto- or paracrine signals. Together, the evidence from the literature and our current findings highlight the importance of purine metabolism in adrenal (patho-) physiology. We appreciate that the direct link and causal relationship between KCNJ5-activating mutations and purine-dependent signaling in APAs remain uncertain and will require more directed mechanistic studies.

It is also noteworthy that lower amounts of 1-phosphatidyl-D-myo-inositol, which is generated from 1-phosphatidyl-D-myo-inositol-5-phosphate via the enzyme 1-phosphatidyl-D-myo-inositol-5-phosphate 4-kinase (PIP4K II), is evident in KCNJ5-mutated APAs. Because negative effects of PIP4Ks on PI3K/Akt signaling have been suggested in previous studies $(33,34)$, lower activity of PIP4K II indicates enhanced Akt activity in KCNJ5-mutated APAs. This finding is in accordance with a previous study, which reported that the $\mathrm{PI}$ K/Akt signaling is activated in some APAs while no genotype/phenotype correlation was provided (35).

As derivatives of cortisol, 18-hydroxycortisol and 18-oxocortisol, summarized as 18-hybrid steroids, have been implicated in the steroidogenic profile of patients with PA. Previous studies have shown that CYP11B2 can convert 11-deoxycortisol efficiently to 18-hydroxycortisol and 18-oxocortisol, while CYP11B1 can synthesize only 18-hydroxycortisol (reviewed in ref. 36). High plasma levels of 18-oxocortisol and 18-hydroxycortisol have been identified in patients with KCNJ5-mutated APAs and have been shown to be predictive for the presence of this mutation (14). Our results provide data on increased intratumor content of 18-oxocortisol and 18-hydroxycortisol in KCNJ5-mutated APAs. Regarding the relationships between steroidogenic enzymes and steroid metabolites, significant inverse correlations between staining intensities of CYP11B1 and staining intensities of 18-hybrid steroids were evident independent of the somatic genotype. Low expression of CYP11B1 may expose CYP11B2 to higher levels of 11-deoxycortisol and allow for efficient conversion toward 18-hybrid steroids. Furthermore, our analyses suggest a contribution of CYP17A1 to the production of 18-oxocortisol. Considering that CYP17A1 generates upstream substrates of 11-deoxycortisol, relationships between higher staining intensities of CYP17A1 and abundance of 18-oxocortisol were expected. Surprisingly, our analysis revealed that positive correlations between CYP17A1 and 18-oxocortisol are present only in tissues with a lower-than-median staining intensity of CYP17A1. These findings might thereby indicate a threshold, 
below which the amount of CYP17A1 can affect the production of 18-oxocortisol. On the other hand, our findings indicate that CYP11B2 expression levels in APAs are not significantly correlated with the production of 18-oxocortisol and 18-hydroxycortisol. One explanation of this finding is that all APA tissues are characterized by high CYP11B2 expression levels above a threshold, which would be critical to detect any difference in 18 steroid levels. Current MALDI-MSI techniques are restricted to a subset of steroids that can be identified and quantified. Future adaptations of analytical protocols would be necessary to overcome these limitations and to provide further insights into steroidogenesis in a spatial manner.

The power of digital image analysis provided us findings about relationships between clinical outcome and tumor metabolite content and steroidogenic enzyme expression in APA tissues. It is therefore the first report to our knowledge that provides a correlation of 18-oxocortisol tumor content and CYP11B1 expression levels with outcome independent of clinical parameters, such as age at adrenalectomy (37). Significant inverse correlation between the intensities of 18-oxocortisol and CYP11B1 may explain in part the correlation between metabolite profiles and clinical parameters. For example, an inverse correlation of 24-hour urinary free cortisol and the intensity of 18-oxocortisol might be due to the abundance of CYP11B1, which promotes cortisol production in APA tissues. Two previous studies revealed that glucocorticoid cosecretion in APAs is frequently found, and several surrogate parameters of metabolic risk were correlated with glucocorticoid secretion, which suggested the concept of "Connshing" syndrome $(15,38)$. Our findings showed the importance of distribution of CYP11B1 in tumor tissues, not that of CYP11B2, with respect to clinical outcome and provided new insights into this concept. Overall, our findings suggest that information on tumor metabolites and steroidogenic enzyme expression could aid the prediction of long-term clinical outcome and therefore might help define the intensity of clinical follow-up and indication of accompanying medical intervention.

The present study indicates distinct metabolomic profiles of APAs with regard to mutation status between $K C N J 5$ and $C A C N A 1 D$. In addition, we reveal an inverse correlation between 18-oxocortisol and 18-hydroxycortisol and CYP11B1 expression. The impact of 18-oxocortisol and CYP11B1 on clinical outcome is demonstrated, which provides potentially unprecedented insights into the pathophysiology, clinical features, and steroidogenesis of APAs.

\section{Methods}

\section{Patient cohort}

The study cohort comprised 136 patients with unilateral PA, who had been diagnosed and surgically treated at a referral center (Klinikum der Universität München, Munich, Germany). The diagnosis of PA was established according to current Endocrine Society Guidelines (39). Baseline and follow-up clinical information was collected prospectively as part of the German Conn registry. Specific details for PA diagnosis and adrenal venous sampling procedures have been described elsewhere (40).

\section{DNA sequencing}

Detection of somatic mutations in APAs. In 45 cases, exome sequencing of frozen tumor samples was performed as described previously (10). From 87 samples, targeted sequencing of APA-related genes (KCNJ5, ATP1A1, ATP2B3, CACNA1D, CTNNB1, and PRKACA) was performed following polymerase chain reaction and direct sequencing. Detailed methods, including primer information, have been described previously $(10,23,41)$.

Tissue microarrays, IHC, and digital image analysis. Tissue microarrays were constructed by sampling of 3 tumor tissue cores (1.0 $\mathrm{mm}$ in diameter) as previously described (42). Paraffin-embedded tissue blocks were used upon selection on the basis of representative H\&E-stained tissue sections of each case by the same pathologist.

The technical construction of the tissue microarrays was performed on a manual tissue array device (AlphaMetrix Biotech). A semiautomated rotation microtome (Microm HM 355S, Thermo Fisher Scientific) was used to cut 3- $\mu \mathrm{m}$ tissue microarray sections that were transferred to glass slides for IHC and $\mathrm{H} \& \mathrm{E}$ staining. IHC staining was performed under standardized conditions on a Discovery XT automated stainer (Ventana Medical Systems, Roche Diagnostics) using monoclonal antibodies directed against human (h) CYP11B1, CYP11B2, CYP17A1 (43), and HSD3B1 (22), developed by one of the co-authors (CGS). Slides were incubated with primary antibodies (rat monoclonal anti-hCYP11B1 [1:20], mouse monoclonal anti-hCYP11B2 [1:100], mouse monoclonal anti-17 $\alpha$-hydroxylase [1:500], mouse monoclonal 
anti-hHSD3B1 [1:500], or rabbit polyclonal anti-hHSD3B2 [1:250] (Abcam, Ab154385), in Dako REAL antibody dilution, Agilent) and detected by the Discovery DAB Map Kit (Ventana Medical Systems, Roche Diagnostics), including incubation with respective secondary antibodies (against CYP11B2, HSD3B1, and HSD3B2: antimouse and antirabbit, ready-to-use universal secondary antibody, 760-4205, Ventana Medical Systems, Roche Diagnostics; against CYP11B1: antirat, ready-to-use universal secondary antibody, BA-4000, Vector Laboratories).

Following H\&E staining and IHC, tissue microarray slides were scanned at $\times 20$ original objective magnification using a digital Mirax Desk slide scanner (Carl Zeiss Microscopy $\mathrm{GmbH}$ ) before importing into the image analysis software Definiens Developer XD2 (Definiens AG), as described previously (44). Tumor areas were manually annotated, and a rule set was defined to detect and quantify morphometry data, including color and shape parameters of nucleus and cytoplasm from H\&E staining, and staining intensities from IHC in the annotated tumor area, with operators blinded with regard to corresponding clinical annotations.

Tissue sectioning and matrix application. TMA samples were sectioned with a thickness of $4 \mu \mathrm{m}$ and water bath-mounted onto indium tin oxide-coated glass slides (Bruker Daltonik GmbH). FFPE sections were incubated for 1 hour at $70^{\circ} \mathrm{C}$, deparaffinized in xylene $(2 \times 8 \mathrm{~min})$, and air-dried. For MALDI-MSI of endogenous metabolites, the matrix solution was $10 \mathrm{mg} / \mathrm{ml}$ 9-aminoacridine hydrochloride monohydrate matrix (9-AA) (MilliporeSigma Chemie $\mathrm{GmbH}$ ) in water/methanol 30:70 (v/v). 9-AA was chosen as a matrix because it is known to exhibit very few matrix-derived interferences in the low-mass range and to achieve high sensitivity and high linearity in negative ion mode for a wide range of low-molecular-weight metabolites (45-49). The matrix solution was sprayed on the tissue sections using a SunCollect automatic sprayer (SunChrom) at room temperature. The matrix application was performed at flow rates of 10, 20, 30 , and $40 \mu 1 / \mathrm{min}$, respectively, for the first 4 layers. The other 4 layers were performed at $40 \mu 1 / \mathrm{min}$.

MALDI-MSI measurement and metabolite annotation. Following tissue sample preparation, the MALDI-MSI measurement was performed on a Bruker Solarix 7T FT-ICR-MS (Bruker Daltonics). The MALDI-MSI data were acquired over a mass range of $m / z 50-1000$. Mass imaging data were acquired in negative ionization mode with $70 \mu \mathrm{m}$ spatial resolution using 50 laser shots at a frequency of $500 \mathrm{~Hz}$. After the MALDI-MSI measurement, the acquired data from the tissue samples underwent spectra processing in FlexImaging v. 4.2 (Bruker Daltonics). Following the MALDI imaging, matrix was removed with 70\% ethanol. Tissue sections were stained with H\&E and scanned with a digital Mirax Desk slide scanner (Carl Zeiss Microscopy $\mathrm{GmbH}$ ).

Metabolite annotation was performed by matching accurate mass with databases as previously described (49-51) (mass accuracy $\leq 4$ ppm, METLIN, http://metlin.scripps.edu/; the Human Metabolome Database, http://www.hmdb.ca; ref. 52). Pathway analysis was applied to identify discriminative features of mutation status of APAs. The Kyoto Encyclopedia of Genes and Genomes database (http:// www.genome.jp/kegg/) and MetaboAnalyst 3.0 (http://www.metaboanalyst.ca) were used.

\section{Statistics}

Metabolomic analysis. MATLAB R2014b (v.7.10.0, MathWorks Inc.) was used as the MALDI spectral preprocessing tool for the subsequent bioinformatics data analysis. Peak picking was performed as described in detail previously (50). Briefly, the parameters of peak picking included $\mathrm{m} / z 0.0005$ minimal peak width, signal-tonoise threshold of 2 , and intensity threshold of $0.01 \%$. Isotopes were automatically identified and excluded. The list of $m / z$ species with respective intensities for ROIs were uploaded to MetaboAnalyst 3.0 (http://www. metaboanalyst.ca) (53) and processed with a mass tolerance of $\mathrm{m} / z$ 0.0001. Metabolites present in more than $80 \%$ of patients were extracted as matrix cleaning and used for heatmap-based clustering and ortho-PLSDA, which were created with MetaboAnalyst, and data were exported as Figure 2B.

Correlation within tissue-derived parameters and clinical annotations. Statistical comparisons between morphometry or metabolome and genotypes were performed with Mann-Whitney $U$ test or Kruskal-Wallis test, and $P$ values were adjusted with the Benjamini-Hochberg method. To calculate the detailed relationship between profiles of tissue-derived parameters and clinical annotations, staining intensities from IHC data and peak intensities from metabolites (18-oxocortisol and 18-hydroxycortisol) of 3 cores from each tumor were classified into highest, median, and lowest intensity, and the median intensity of 3 cores from each tumor was used for the analysis with Mann-Whitney $U$ test, Kruskal-Wallis test, or Spearman's rank correlation coefficient. Spearman's correlation coefficients are denoted as $r_{s} . P<0.05$ was considered significant. 
Correlations within IHC data and between IHC data and metabolome profiles were calculated at each core to appreciate potential intratumor heterogeneity of steroidogenesis. Further statistical testing for associations of profiles of IHC and metabolome to the clinical outcome was conducted using logistic regression. Covariates from clinical parameters were chosen based on Table 1 and analyzed, including profiles of IHC or metabolome data using backward stepwise selection. All calculations were performed using R 3.4.3.

\section{Study approval}

The study was approved by the institutional ethics committee of the Klinikum der Universität München. All patients provided their written informed consent before participating in the study.

\section{Author contributions}

YR, MR, and FB designed the research. YR, T. Knösel, T. Kirchner, TAW, MR, and FB collected samples and clinical data from patients. YR and TMS performed DNA sequencing. MM, YR, T. Kunzke, NS, AF, PL, CGS, TAW, AKW, and FB performed morphometry, immunostaining, and MALDI-FT-ICR MSI analysis and performed statistical analysis of results. As a pathologist, AKW selected areas for the analysis on the basis of representative H\&E-stained tissue sections. MM drafted the manuscript, and all authors contributed to writing the manuscript and approved the version to be published.

\section{Acknowledgments}

We would like to thank Ulrike Buchholz, Claudia-Mareike Pflüger, Andreas Voss, Jana Drechsler, Brigitte Mauracher, Petra Rank, and Christina Brugger for excellent technical assistance. This study was supported by the Deutsche Forschungsgemeinschaft (German Research Foundation) project number 314061271 - TRR 205 to FB, MR, TAW, and AKW. MR was supported by Else Kröner-Fresenius Stiftung (2013_A182 and 2015_A171) and the European Research Council under the European Union's Horizon 2020 research and innovation program (grant agreement 694913). CGS was supported by the National Institute of General Medical Sciences grant U54 GM115428. YR was supported by the Friedrich Baur Stiftung, grant number 46/16, "Genetics and Tissue-based Metabolomics of Aldosterone Producing Adenoma." MM was supported by Japan Heart Foundation/Bayer Yakuhin Research Grant Abroad and a postdoctoral fellowship of the Uehara Memorial Foundation.

Address correspondence to: Felix Beuschlein, Klinik für Endokrinologie, Diabetologie und Klinische Ernährung, Universitätsspital Zürich, Raemistrasse 100, CH-8091 Zurich, Switzerland. Phone: 41.44.255.36.25; Email: felix.beuschlein@usz.ch.

1. Hannemann A, Wallaschofski H. Prevalence of primary aldosteronism in patient's cohorts and in population-based studies - a review of the current literature. Horm Metab Res. 2012;44(3):157-162.

2. Ohno Y, et al. Prevalence of cardiovascular disease and its risk factors in primary aldosteronism: a multicenter study in Japan. Hypertension. 2018;71(3):530-537.

3. Williams TA, et al. Outcomes after adrenalectomy for unilateral primary aldosteronism: an international consensus on outcome measures and analysis of remission rates in an international cohort. Lancet Diabetes Endocrinol. 2017;5(9):689-699.

4. Nishimoto K, et al. Adrenocortical zonation in humans under normal and pathological conditions. J Clin Endocrinol Metab. 2010;95(5):2296-2305.

5. Gomez-Sanchez CE, et al. Development of monoclonal antibodies against human CYP11B1 and CYP11B2. Mol Cell Endocrinol. 2014;383(1-2):111-117.

6. Zennaro MC, Jeunemaitre X, Boulkroun S. Integrating genetics and genomics in primary aldosteronism. Hypertension. 2012;60(3):580-588.

7. Murakami M, et al. Integration of transcriptome and methylome analysis of aldosterone-producing adenomas. Eur J Endocrinol. 2015;173(2):185-195.

8. Murakami M, et al. Molecular characteristics of the KCNJ5 mutated aldosterone-producing adenomas. Endocr Relat Cancer. 2017;24(10):531-541.

9. Choi $\mathrm{M}$, et al. $\mathrm{K}^{+}$channel mutations in adrenal aldosterone-producing adenomas and hereditary hypertension. Science 2011;331(6018):768-772.

10. Beuschlein F, et al. Somatic mutations in ATP1A1 and ATP2B3 lead to aldosterone-producing adenomas and secondary hypertension. Nat Genet. 2013;45(4):440-444.

11. Azizan EA, et al. Somatic mutations in ATP1A1 and CACNA1D underlie a common subtype of adrenal hypertension. Nat Genet. 2013;45(9):1055-1060.

12. Scholl UI, et al. Recurrent gain of function mutation in calcium channel CACNA1H causes early-onset hypertension with primary aldosteronism. Elife. 2015;4:e06315. 
13. Åkerström T, et al. Activating mutations in CTNNB1 in aldosterone producing adenomas. Sci Rep. 2016;6:19546.

14. Williams TA, et al. Genotype-specific steroid profiles associated with aldosterone-producing adenomas. Hypertension. 2016;67(1):139-145

15. Arlt W, et al. Steroid metabolome analysis reveals prevalent glucocorticoid excess in primary aldosteronism. JCI Insight. 2017;2(8):93136.

16. Walch A, Rauser S, Deininger SO, Höfler H. MALDI imaging mass spectrometry for direct tissue analysis: a new frontier for molecular histology. Histochem Cell Biol. 2008;130(3):421-434.

17. Sun N, et al. High-resolution tissue mass spectrometry imaging reveals a refined functional anatomy of the human adult adrenal gland. Endocrinology. 2018;159(3):1511-1524.

18. Nakamura Y, Felizola SJ, Satoh F, Konosu-Fukaya S, Sasano H. Dissecting the molecular pathways of primary aldosteronism. Pathol Int. 2014;64(10):482-489.

19. Neville AM, O'Hare MJ. Histopathology of the human adrenal cortex. Clin Endocrinol Metab. 1985;14(4):791-820.

20. Monticone S, et al. Immunohistochemical, genetic and clinical characterization of sporadic aldosterone-producing adenomas. Mol Cell Endocrinol. 2015;411:146-154.

21. Tan GC, et al. Aldosterone-producing adenomas: histopathology-genotype correlation and identification of a novel $C A C N A 1 D$ mutation. Hypertension. 2017;70(1):129-136.

22. Gomez-Sanchez CE, Lewis M, Nanba K, Rainey WE, Kuppusamy M, Gomez-Sanchez EP. Development of monoclonal antibodies against the human 3ß-hydroxysteroid dehydrogenase/isomerase isozymes. Steroids. 2017;127:56-61.

23. Fernandes-Rosa FL, et al. Genetic spectrum and clinical correlates of somatic mutations in aldosterone-producing adenoma. Hypertension. 2014;64(2):354-361.

24. Åkerström T, et al. Novel somatic mutations and distinct molecular signature in aldosterone-producing adenomas. Endocr Relat Cancer. 2015;22(5):735-744.

25. Azizan EA, et al. Somatic mutations affecting the selectivity filter of KCNJ5 are frequent in 2 large unselected collections of adrenal aldosteronomas. Hypertension. 2012;59(3):587-591.

26. Boulkroun S, et al. Prevalence, clinical, and molecular correlates of KCNJ5 mutations in primary aldosteronism. Hypertension. 2012;59(3):592-598.

27. Oßwald A, et al. Lack of influence of somatic mutations on steroid gradients during adrenal vein sampling in aldosterone-producing adenoma patients. Eur J Endocrinol. 2013;169(5):657-663.

28. Rossi GP, et al. KCNJ5 gene somatic mutations affect cardiac remodelling but do not preclude cure of high blood pressure and regression of left ventricular hypertrophy in primary aldosteronism. J Hypertens. 2014;32(7):1514-21.

29. Pedley AM, Benkovic SJ. A new view into the regulation of purine metabolism: The Purinosome. Trends Biochem Sci. 2017;42(2):141-154

30. Kawamura M, Matsui T, Niitsu A, Kondo T, Ohno Y, Nakamichi N. Extracellular ATP stimulates steroidogenesis in bovine adrenocortical fasciculata cells via P2 purinoceptors. Jpn J Pharmacol. 1991;56(4):543-545.

31. Xu L, Enyeart JJ. Purine and pyrimidine nucleotides inhibit a noninactivating $\mathrm{K}^{+}$current and depolarize adrenal cortical cells through a G protein-coupled receptor. Mol Pharmacol. 1999;55(2):364-376.

32. Nishi H, Arai H, Momiyama T. NCI-H295R, a human adrenal cortex-derived cell line, expresses purinergic receptors linked to $\mathrm{Ca}^{2+}$-mobilization/influx and cortisol secretion. PLoS One. 2013;8(8):e71022.

33. Carricaburu V, et al. The phosphatidylinositol (PI)-5-phosphate 4-kinase type II enzyme controls insulin signaling by regulating PI-3,4,5-trisphosphate degradation. Proc Natl Acad Sci U S A. 2003;100(17):9867-9872.

34. Lamia KA, Peroni OD, Kim YB, Rameh LE, Kahn BB, Cantley LC. Increased insulin sensitivity and reduced adiposity in phosphatidylinositol 5-phosphate 4-kinase $\beta^{-/-}$mice. Mol Cell Biol. 2004;24(11):5080-5087.

35. Su H, et al. The PI3K/AKT/mTOR signaling pathway is overactivated in primary aldosteronism. PLoS One. 2013;8(4):e62399.

36. Lenders JWM, Williams TA, Reincke M, Gomez-Sanchez CE. Diagnosis of endocrine disease: 18-oxocortisol and 18-hydroxycortisol: is there clinical utility of these steroids? Eur J Endocrinol. 2018;178(1):R1-R9.

37. Yang Y, Reincke M, Williams TA. Treatment of unilateral PA by adrenalectomy: potential reasons for incomplete biochemical cure. Exp Clin Endocrinol Diabetes. 2019;127(2-03):100-108.

38. Gerards J, et al. Impaired glucose metabolism in primary aldosteronism is associated with cortisol co-secretion. J Clin Endocrinol Metab. 2019; 104(8):3192-3202.

39. Funder JW, et al. Case detection, diagnosis, and treatment of patients with primary aldosteronism: an endocrine society clinical practice guideline. J Clin Endocrinol Metab. 2008;93(9):3266-3281.

40. Riester A, et al. Age below 40 or a recently proposed clinical prediction score cannot bypass adrenal venous sampling in primary aldosteronism. J Clin Endocrinol Metab. 2014;99(6):E1035-E1039.

41. Rhayem Y, et al. PRKACA somatic mutations are rare findings in aldosterone-producing adenomas. J Clin Endocrinol Metab. 2016;101(8):3010-3017.

42. Rauser S, et al. Significance of HER2 low-level copy gain in Barrett's cancer: implications for fluorescence in situ hybridization testing in tissues. Clin Cancer Res. 2007;13(17):5115-5123.

43. Meyer LS, et al. Immunohistopathology and steroid profiles associated with biochemical outcomes after adrenalectomy for unilateral primary aldosteronism. Hypertension. 2018;72(3):650-657.

44. Feuchtinger A, et al. Image analysis of immunohistochemistry is superior to visual scoring as shown for patient outcome of esophageal adenocarcinoma. Histochem Cell Biol. 2015;143(1):1-9.

45. Edwards JL, Kennedy RT. Metabolomic analysis of eukaryotic tissue and prokaryotes using negative mode MALDI time-offlight mass spectrometry. Anal Chem. 2005;77(7):2201-2209.

46. Shroff R, Muck A, Svatos A. Analysis of low molecular weight acids by negative mode matrix-assisted laser desorption/ionization time-of-flight mass spectrometry. Rapid Commun Mass Spectrom. 2007;21(20):3295-3300.

47. Miura D, Fujimura Y, Tachibana H, Wariishi H. Highly sensitive matrix-assisted laser desorption ionization-mass spectrometry for high-throughput metabolic profiling. Anal Chem. 2010;82(2):498-504.

48. Miura D, et al. Ultrahighly sensitive in situ metabolomic imaging for visualizing spatiotemporal metabolic behaviors. Anal 
Chem. 2010;82(23):9789-9796.

49. Sun N, et al. High-resolution metabolite imaging of light and dark treated retina using MALDI-FTICR mass spectrometry. Proteomics. 2014;14(7-8):913-923.

50. Buck A, et al. High-resolution MALDI-FT-ICR MS imaging for the analysis of metabolites from formalin-fixed, paraffin-embedded clinical tissue samples. J Pathol. 2015;237(1):123-132.

51. Aichler M, et al. N-acyl taurines and acylcarnitines cause an imbalance in insulin synthesis and secretion provoking $\beta$ cell dysfunction in type 2 diabetes. Cell Metab. 2017;25(6):1334-1347.e4.

52. Wishart DS, et al. HMDB: the Human Metabolome Database. Nucleic Acids Res. 2007;35(Database issue):D521-D526

53. Xia J, Wishart DS. MetPA: a web-based metabolomics tool for pathway analysis and visualization. Bioinformatics. 2010;26(18):2342-2344. 